\title{
Hemolytic uremic syndrome and encephalopathy from Shiga toxin-producing Escherichia coli
}

\author{
Toru Ishihara MD, Hideki Ozawa MD \\ - Cite as: CMAJ 2019 July 22;191:E817. doi: 10.1503/cmaj.190155
}

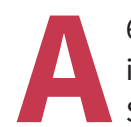

60-year-old Japanese woman was admitted to hospital for impaired consciousness and convulsive status epilepticus. She had received supportive care for bloody diarrhea at a nearby hospital 9 days earlier. Blood test results at admission were as follows: white blood cell count, 43.5 (normal 4.0-10.0) $\times 10^{9} / \mathrm{L}$; hemoglobin, 99 (normal 120-150) g/L; platelet count, 23 (normal 150-400) $\times 10^{9} / \mathrm{L}$; lactate dehydrogenase, 1306 (normal 110-240) U/L; and creatinine, 137.9 (normal 50-90) $\mu \mathrm{mol} / \mathrm{L}$. The other blood test results were unremarkable. Colonoscopy showed mucosal hyperemia and edema, and computed tomography showed generalized thickening of the colonic wall (Figure 1A, B). A stool culture tested positive for Shiga toxin-producing Escherichia coli 0157 . Diffusion-weighted and $T_{2}$-weighted fluid-attenuated inversion recovery magnetic resonance imaging (MRI) showed symmetric high signals in the thalamus (Figure 1C), basal ganglia, insular cortex, external capsule and subcortical occipital lobe (Figure 1D). High signals were observed in the pons and periphery of the fourth ventricle (see video, Appendix 1, available at www. cmaj.ca/lookup/suppl/doi:10.1503/cmaj.190155/-/DC1). The patient was diagnosed with hemolytic uremic syndrome and encephalopathy associated with Shiga toxin-producing E. coli. She was treated with anti-epileptic drugs and ventilator management in the intensive care unit (ICU); given the severity of her neurologic symptoms, she received hemodialysis and plasma exchange. Her general condition improved, and she was discharged from the ICU on day 13.

The incidence of infection with Shiga toxin-producing E. coli is estimated to be about 2.8 million acute cases annually, with about 4000 cases complicated by hemolytic uremic syndrome. ${ }^{1}$ The transmission route is oral, through consumption of contaminated food and water. Involvement of the central nervous system affects mortality. ${ }^{2}$ Abnormal findings associated with encephalopathy in hemolytic uremic syndrome are usually found bilaterally, in the thalamus and pons. ${ }^{3}$ Our patient showed a wide range of abnormal findings, and the differential diagnosis included toxic metabolic encephalopathy (e.g., from hypoxia or hypoglycemia). Brain MRI does not provide a definitive diagnosis but is useful to diagnose encephalopathy and select treatment. Timely plasma exchange may be helpful to treat encephalopathy associated with Shiga toxin-producing E. coli. ${ }^{4}$

A video of magnetic resonance imaging of the patient's brain is available in Appendix 1, at www.cmaj.ca/lookup/ suppl/doi:10.1503/cmaj.190155/-/DC1.
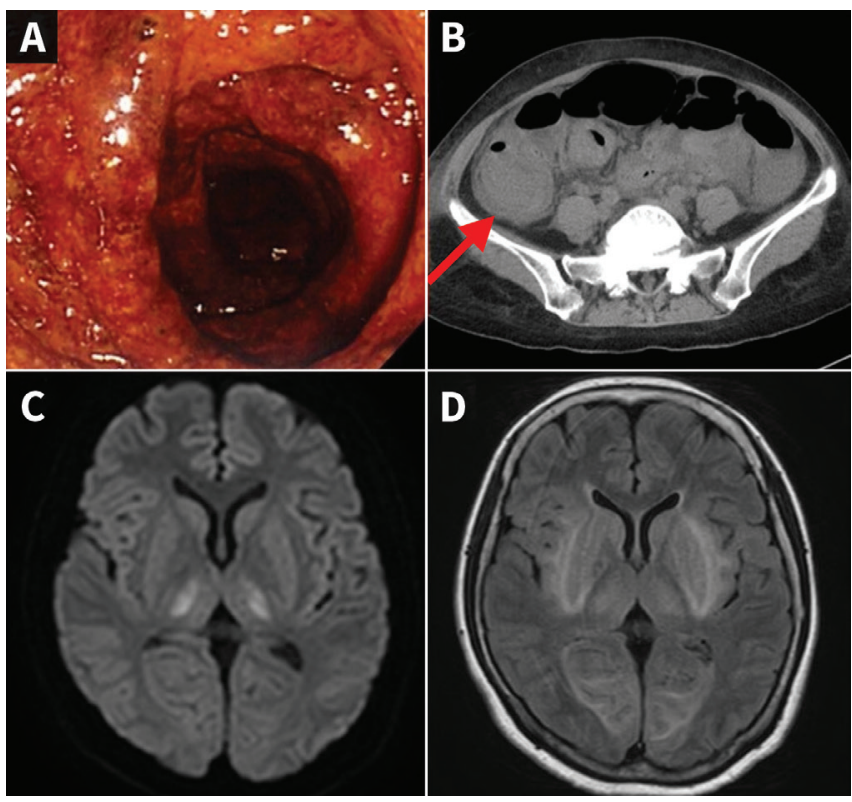

Figure 1: (A) Colonoscopy image in a 60-year-old woman with hemolytic uremic syndrome from Shiga toxin-producing Escherichia coli, showing mucosal hyperemia, marked edema and red areas of hemorrhage, indicating hemorrhagic colitis. (B) Computed tomography image showing generalized thickening of the colonic wall, particularly in the ascending colon (target sign, arrow). (C, D) Diffusion-weighted and $T_{2}$-weighted fluid-attenuated inversion magnetic resonance images of the brain showing symmetric high signals indicating encephalopathy associated with hemolytic uremic syndrome.

\section{References}

1. Majowicz SE, Scallan E, Jones-Bitton A, et al. Global incidence of human Shiga toxin-producing Escherichia coli infections and deaths: a systematic review and knowledge synthesis. Foodborne Pathog Dis 2014;11:447-55.

2. Torres AG, Amaral MM, Bentancor L, et al. Recent advances in shiga toxinproducing Escherichia coli research in Latin America. Microorganisms 2018;6:E100.

3. Magnus T, Röther J, Simova $O$, et al. The neurological syndrome in adults during the 2011 northern German E. coli serotype 0104:H4 outbreak. Brain 2012;135:1850-9.

4. Schwartz J, Padmanabhan A, Aqui N, et al. Guidelines on the use of therapeutic apheresis in clinical practice-evidence-based approach from the Writing Committee of the American Society for Apheresis: the seventh special issue. J Clin Apher 2016;31:149-62.

\section{Competing interests: None declared.}

This article has been peer reviewed.

The authors have obtained patient consent.

Affiliation: Division of General Internal Medicine, Tokai University School of Medicine, Kanagawa, Japan

Correspondence to: Toru Ishihara, it735130@tsc.u-tokai.ac.jp 